\title{
On the solutions of boundary value problems
}

\author{
Ali Akgül ${ }^{a^{*}}$, Mir Sajjad Hashemi ${ }^{b}$ and Negar Seyfi ${ }^{b}$ \\ ${ }^{a}$ Siirt University, Art and Science Faculty, Department of Mathematics, Siirt, Turkey \\ ${ }^{b}$ Department of Mathematics, Basic Science Faculty, University of Bonab, Bonab, Iran \\ aliakgul00727@gmail.com, hashem_math396@yahoo.com,n.seifi2017@gmail.com
}

\begin{tabular}{|c|c|}
\hline ARTICLE INFO & ABSTRACT \\
\hline Article History: & \multirow{5}{*}{$\begin{array}{l}\text { We investigate the nonlinear boundary value problems by reproducing kernel } \\
\text { Hilbert space technique in this paper. We construct some reproducing kernel } \\
\text { Hilbert spaces. We define a bounded linear operator to obtain the solutions of } \\
\text { the problems. We demonstrate our numerical results by some tables. We com- } \\
\text { pare our numerical results with some results exist in the literature to present } \\
\text { the efficiency of the proposed method. }\end{array}$} \\
\hline Received 26 August 2020 & \\
\hline Accepted 05 January 2021 & \\
\hline Available 12 May 2021 & \\
\hline $\begin{array}{l}\text { Keywords: } \\
\text { Reproducing kernel Hilbert }\end{array}$ & \\
\hline
\end{tabular}

space method

Boundary value problems

Bounded linear operator

Reproducing kernel functions

AMS Classification 2010:

47B32; $34 B 05$

\section{Introduction}

We investigate the following boundary value problems by reproducing kernel method.

$$
\left(p(x) y^{\prime}\right)^{\prime}=f(x, y)
$$

subject to the boundary values

$$
y(0)=A, \quad y(1)=B .
$$

Reproducing kernel space is a special Hilbert space. Many problems have been investigated by reproducing kernel Hilbert space method in the literature.

Safari et al. [1] have investigated the rainfallrunoff modeling through regression in the reproducing kernel Hilbert space algorithm. Najafi et al. 2] have worked on the combining fractional differential transform method and reproducing kernel Hilbert space method to solve fuzzy impulsive fractional differential equations. Sahihi et al. 3 have searched the system of second-order boundary value problems using a new algorithm based on the reproducing kernel Hilbert space. Agud et al. 4 have investigated the weighted p-regular kernels for reproducing kernel Hilbert spaces. Mundayadan et al. [5] have studied on the linear dynamics in the reproducing kernel Hilbert spaces. Arqub et al. [6] have constructed the modulation of reproducing kernel technique successfully. Emamjome et al. [7] have presented the reproducing kernel pseudospectral technique in details. Foroutan et al. 8 have investigated this technique for the nonlinear three-point boundary value problems. Akgül et al. [9] have worked on the representation for the reproducing kernel Hilbert space method for a nonlinear system. Allahviranloo et al. 14] have investigated the reproducing kernel method to solve parabolic partial differential equations with nonlocal conditions. For more details see [15 23].

We organize our manuscript as: We construct the reproducing kernel Hilbert spaces in Section 2. We apply the reproducing kernel method in this section. We demonstrate the numerical results in Section 3. We give the conclusion in the last section. 


\section{Reproducing kernel Hilbert spaces}

We define the reproducing kernel Hilbert spaces and find some reproducing kernel functions in these spaces in this section.

Definition 1. Let $(H,\langle\cdot, \cdot\rangle)$ be a Hilbert space of real functions defined on a nonempty set $E$. A function $K: E \times E \rightarrow \mathbb{R}$ is called a reproducing kernel for $H$ if and only if

(a) $K(\cdot, z) \in H$ for each fixed $z \in E$,

(b) $\langle\varphi, K(\cdot, z)\rangle=\varphi(z)$ for all $z \in E$ and all $\varphi \in H$.

We will refer to such a Hilbert space $H$ for which there exists a reproducing kernel function $K$ as a reproducing kernel Hilbert space.

Condition (b) is called "the reproducing property" of the kernel $K$ because the value of an arbitrary function $\varphi \in H$ at an arbitrary point $z \in E$ is reproduced by the inner product of $\varphi$ with $K(\cdot, z)$.

Definition 2. $\mathcal{W}_{2}^{1}[0,1]$ is given as:

$\mathcal{W}_{2}^{1}[0,1]=\left\{y: y \in A C[0,1]\right.$ and $\left.y^{\prime} \in L^{2}[0,1]\right\}$, with

$$
\begin{array}{r}
\langle y, g\rangle_{\mathcal{W}_{2}^{1}}=\int_{0}^{1}\left[y(x) g(x)+y^{\prime}(x) g^{\prime}(x)\right] \mathrm{d} x, \\
y, g \in \mathcal{W}_{2}^{1}[0,1],
\end{array}
$$

and

$$
\|y\|_{\mathcal{W}_{2}^{1}}=\sqrt{\langle y, y\rangle_{\mathcal{W}_{2}^{1}}}, \quad y \in \mathcal{W}_{2}^{1}[0,1]
$$

as the inner product and the norm in $\mathcal{W}_{2}^{1}[0,1]$ respectively. Reproducing kernel function $T_{x}(y)$ of $\mathcal{W}_{2}^{1}[0,1]$ is presented as:

$T_{x}(y)=\frac{1}{2 \sinh (1)}[\cosh (x+y-1)+\cosh (|x-y|-1)]$

Definition 3. The space ${ }^{o} \mathcal{W}_{2}^{3}[0,1]$ is given by

$$
\begin{aligned}
& { }^{o} \mathcal{W}_{2}^{3}[0,1]=\left\{y \in A C[0,1]: y^{\prime}, y^{\prime \prime} \in A C[0,1],\right. \\
& \left.y^{(3)} \in L^{2}[0,1], y(0)=0=y(1)\right\} . \\
& \langle y, v\rangle_{o{ }^{\circ} \mathcal{W}_{2}^{3}[0,1]}=y(0) v(0)+y^{\prime}(0) v^{\prime}(0)+y(1) v(1) \\
& +\int_{0}^{1} y^{(3)}(x) v^{(3)}(x) \mathrm{d} x, \\
& y, v \in{ }^{o} \mathcal{W}_{2}^{3}[0,1] \text {, }
\end{aligned}
$$

and

$$
\|y\|_{{ }_{o} \mathcal{W}_{2}^{3}[0,1]}=\sqrt{\langle y, y\rangle_{o \mathcal{W}_{2}^{3}[0,1]}}, \quad y \in{ }^{o} \mathcal{W}_{2}^{3}[0,1]
$$

are the inner product and the norm in ${ }^{\circ} \mathcal{W}_{2}^{3}[0,1]$ respectively.

Theorem 1. Reproducing kernel function $y(x)$ of ${ }^{\circ} \mathcal{W}_{2}^{3}[0,1]$ is given as

$$
\mathcal{R}_{\xi}(x)=\left\{\begin{array}{l}
-x^{2} \xi-x \xi^{2}+x \xi-\frac{1}{120} x^{2} \xi^{2}+\frac{21}{20} x^{2} \xi^{2} \\
+\frac{1}{24} x^{2} \xi^{4}-\frac{1}{12} x^{2} \xi^{3} \\
+\frac{1}{24} x^{4} \xi^{2}-\frac{1}{24} x^{4} \xi-\frac{1}{120} x^{5} \xi^{2}+\frac{x^{5}}{120} \\
0 \leq x \leq \xi \leq 1 \\
\\
-\xi^{2} x-\xi x^{2}+\xi x-\frac{1}{120} \xi^{2} x^{2}+\frac{21}{20} \xi^{2} x^{2} \\
+\frac{1}{24} \xi^{2} x^{4}-\frac{1}{12} \xi^{2} x^{3} \\
+\frac{1}{24} \xi^{4} x^{2}-\frac{1}{24} \xi^{4} x-\frac{1}{120} \xi^{5} x^{2}+\frac{\xi^{5}}{120} \\
0 \leq \xi<x \leq 1
\end{array}\right.
$$

\section{Proof. First, let us suppose}

$$
\mathcal{R}_{\xi}(x)= \begin{cases}\sum_{i=1}^{6} c_{i}(\xi) x^{i-1}, & 0 \leq x \leq \xi \leq 1, \\ \sum_{i=1}^{6} d_{i}(\xi) x^{i-1}, & 0 \leq \xi<x \leq 1 .\end{cases}
$$

Then from $y \in{ }^{o} \mathcal{W}_{2}^{3}[0,1]$, we get

$$
\begin{aligned}
& \left\langle y(x), \mathcal{R}_{\xi}(x)\right\rangle_{o_{2} W_{2}^{3}[0,1]}=y(0) \mathcal{R}_{\xi}(0)+y^{\prime}(0) \mathcal{R}_{\xi}^{\prime}(0)+y(1) \mathcal{R}_{\xi}(1) \\
& +\int_{0}^{1} y^{(3)}(x) \frac{\partial^{3} \mathcal{R}_{\xi}(x)}{\partial x^{3}} \mathrm{~d} x \\
& =y(0) \mathcal{R}_{\xi}(0)+y^{\prime}(0) \frac{\partial \mathcal{R}_{\xi}(0)}{\partial x} \\
& +y(1) \mathcal{R}_{\xi}(1)+y^{\prime \prime}(1) \frac{\partial^{3} \mathcal{R}_{\xi}(1)}{\partial x^{3}} \\
& -y^{\prime \prime}(0) \frac{\partial^{3} \mathcal{R}_{\xi}(0)}{\partial x^{3}}-y^{\prime}(1) \frac{\partial^{4} \mathcal{R}_{\xi}(1)}{\partial x^{4}} \\
& +y^{\prime}(0) \frac{\partial^{4} \mathcal{R}_{\xi}(0)}{\partial x^{4}}+y(1) \frac{\partial^{5} \mathcal{R}_{\xi}(1)}{\partial x^{5}}-y(0) \frac{\partial^{5} \mathcal{R}_{\xi}(0)}{\partial x^{5}} \\
& -\int_{0}^{1} y(x) \frac{\partial^{6} \mathcal{R}_{\xi}(x)}{\partial x^{6}} \mathrm{~d} x .
\end{aligned}
$$

Solving the coefficients, we get the reproducing kernel function as: 


$$
\mathcal{R}_{\xi}(x)=\left\{\begin{array}{l}
-x^{2} \xi-x \xi^{2}+x \xi-\frac{1}{120} x^{2} \xi^{2}+\frac{21}{20} x^{2} \xi^{2} \\
+\frac{1}{24} x^{2} \xi^{4}-\frac{1}{12} x^{2} \xi^{3} \\
+\frac{1}{24} x^{4} \xi^{2}-\frac{1}{24} x^{4} \xi-\frac{1}{120} x^{5} \xi^{2}+\frac{x^{5}}{120} \\
0 \leq x \leq \xi \leq 1 \\
-\xi^{2} x-\xi x^{2}+\xi x-\frac{1}{120} \xi^{2} x^{2} \\
+\frac{21}{20} \xi^{2} x^{2}+\frac{1}{24} \xi^{2} x^{4}-\frac{1}{12} \xi^{2} x^{3} \\
+\frac{1}{24} \xi^{4} x^{2}-\frac{1}{24} \xi^{4} x-\frac{1}{120} \xi^{5} x^{2}+\frac{\xi^{5}}{120} \\
0 \leq \xi<x \leq 1 .
\end{array}\right.
$$

\subsection{Solutions in ${ }^{o} \mathcal{W}_{2}^{3}[0,1]$}

We consider the solution of Eq.(1) in the reproducing kernel space ${ }^{o} \mathcal{W}_{2}^{3}[0,1]$ in this section. On defining the operator

$$
\mathcal{L}:{ }^{o} \mathcal{W}_{2}^{3}[0,1] \rightarrow \mathcal{W}_{2}^{1}[0,1],
$$

problem (11) converts as:

$$
\left\{\begin{array}{l}
\mathcal{L} y=\mathfrak{f}(x, u), \quad x \in[0,1], \\
y(0)=A, y(1)=B,
\end{array}\right.
$$

We should homogenize the conditions. Put

$$
u(x)=y(x)+(A-B) x-A,
$$

then we can obtain homogeneous boundary-value conditions of problem (1)

$$
\mathcal{L} u(x)=p^{\prime}(x) u^{\prime}+p(x) u^{\prime \prime} .
$$

With the boundary conditions:

$$
\left\{\begin{array}{l}
\mathcal{L} u=\mathfrak{g}(x, u), \quad x \in[0,1] \\
u(0)=u(1)=0
\end{array}\right.
$$

where

$$
\mathfrak{g}(x, u)=f(x, u)+p^{\prime}(x)(A-B) .
$$

Theorem 2. The operator $\mathcal{L}$ is a bounded linear operator.

Proof. Firstly, we present $\|\mathcal{L} u\|_{\mathcal{W}_{2}^{1}}^{2} \leq \mathcal{M}\|u\|_{{ }^{\circ} \mathcal{W}_{2}^{3}}^{2}$, with $\mathcal{M}>0$. By

(3) and (3), we get

$$
\begin{aligned}
\|\mathcal{L} u\|_{\mathcal{W}_{2}^{1}}^{2} & =\langle\mathcal{L} u, \mathcal{L} u\rangle_{\mathcal{W}_{2}^{1}} \\
& =\int_{0}^{1}\left[((\mathcal{L} u)(x))^{2}+\left((\mathcal{L} u)^{\prime}(x)\right)^{2}\right] \mathrm{d} x .
\end{aligned}
$$

Moreover, by reproducing property we have:

$$
y(x)=\left\langle y(\cdot), \mathcal{R}_{x}(\cdot)\right\rangle_{\circ \mathcal{W}_{2}^{3}} .
$$

Then, we get

$$
\begin{aligned}
\mathcal{L} u(x) & =\left\langle u(\cdot), \mathcal{L R}_{x}(\cdot)\right\rangle_{{ }_{o} \mathcal{W}_{2}^{3}} \\
& =\left\langle u(\cdot),\left(\mathcal{L}_{1}+\mathcal{L}_{2}\right) \mathcal{R}_{x}(\cdot)\right\rangle_{o} \mathcal{W}_{2}^{3} \\
& =\left\langle u(\cdot), \mathcal{L}_{1} \mathcal{R}_{x}(\cdot)\right\rangle_{o} \mathcal{W}_{2}^{3}+\left\langle u(\cdot), \mathcal{L}_{2} \mathcal{R}_{x}(\cdot)\right\rangle_{{ }_{o} \mathcal{W}_{2}^{3}} .
\end{aligned}
$$

With condition to $p(x) \in C^{2}[0,1], \mathcal{M}_{p}=$ $\max \left\{|p(x)|,\left|p^{\prime}(x)\right|,\left|p^{\prime \prime}(x)\right| \quad 0 \leq x \leq 1\right\}$,

$\mathcal{M}_{1}=\max \left\{\frac{\partial}{\partial x} \mathcal{R}_{x}(\xi) \quad 0 \leq \xi \leq 1\right\}$, and $\mathcal{M}_{2}=$ $\max \left\{\frac{\partial}{\partial x^{2}} \mathcal{R}_{x}(\xi) \quad 0 \leq \xi \leq 1\right\}$ then

$$
\begin{aligned}
& |\mathcal{L} u(x)| \leq\|u\|_{{ } \mathcal{W}_{2}^{3}}\left\|\mathcal{L}_{1} \mathcal{R}_{x}\right\|_{{ }^{o} \mathcal{W}_{2}^{3}}+\|u\|_{{ } \mathcal{W}_{2}^{3}}\left\|\mathcal{L}_{2} \mathcal{R}_{x}\right\|_{{ }^{o} \mathcal{W}_{2}^{3}} \\
& =\mathcal{M}_{1} \mathcal{M}_{p}\|u\|_{o_{\mathcal{W}_{2}^{3}}}+\mathcal{M}_{2} \mathcal{M}_{p}\|u\|_{o \mathcal{W}_{2}^{3}} \\
& =\left(\mathcal{M}_{1}+\mathcal{M}_{2}\right) \mathcal{M}_{p}\|u\|_{o_{\mathcal{W}}^{3}}
\end{aligned}
$$

where $\mathcal{M}_{1}>0, \mathcal{M}_{2}>0, \mathcal{M}_{p}>0$. Therefore

$$
\int_{0}^{1}[(\mathcal{L} y)(x)]^{2} \mathrm{~d} x \leq\left(\mathcal{M}_{1}+\mathcal{M}_{2}\right)^{2} \mathcal{M}_{p}^{2}\|u\|_{{ }^{2} \mathcal{W}_{2}^{3}}^{2} .
$$

Also, from

$$
\begin{aligned}
& (\mathcal{L} u)^{\prime}(x)=\left\langle u(\cdot),\left(\mathcal{L} \mathcal{R}_{x}\right)^{\prime}(\cdot)\right\rangle_{{ }_{o} \mathcal{W}_{2}^{3}} \\
& \left.=\left\langle u(\cdot),\left(\mathcal{L}_{1}+\mathcal{L}_{2}\right) \mathcal{R}_{x}\right)^{\prime}(\cdot)\right\rangle_{{ }_{o} \mathcal{W}_{2}^{3}} \\
& =\left\langle u(\cdot),\left(\mathcal{L}_{1} \mathcal{R}_{x}\right)^{\prime}(\cdot)\right\rangle_{{ }_{o \mathcal{W}_{2}^{3}}}+\left\langle u(\cdot),\left(\mathcal{L}_{2} \mathcal{R}_{x}\right)^{\prime}(\cdot)\right\rangle_{{ }_{o} \mathcal{W}_{2}^{3}}
\end{aligned}
$$

we have condition to $p(x) \in C^{2}[0,1]$, $\mathcal{M}_{p}=\max \left\{|p(x)|,\left|p^{\prime}(x)\right|,\left|p^{\prime \prime}(x)\right| 0 \leq x \leq 1\right\}$ $\mathcal{M}_{1}=\max \left\{\frac{\partial}{\partial x} \mathcal{R}_{x}(\xi) \quad 0 \leq \xi \leq 1\right\}, \mathcal{M}_{2}=$ $\max \left\{\frac{\partial}{\partial x^{2}} \mathcal{R}_{x}(\xi) 0 \leq \xi \leq 1\right\}$ and $\mathcal{M}_{3}=$ $\max \left\{\frac{\partial}{\partial x^{2}} \mathcal{R}_{x}(\xi) \quad 0 \leq \xi \leq 1\right\}$ then

$$
\begin{aligned}
\left|(\mathcal{L} u)^{\prime}(x)\right| & \leq\|u\|_{o_{\mathcal{W}}^{3}}\left\|\left(\mathcal{L}_{1} \mathcal{R}_{x}\right)^{\prime}\right\|_{o \mathcal{W}_{2}^{3}} \\
& +\|u\|_{o \mathcal{W}_{2}^{3}}\left\|\left(\mathcal{L}_{2} \mathcal{R}_{x}\right)^{\prime}\right\|_{{ }^{o} \mathcal{W}_{2}^{3}} \\
& =\left(\mathcal{M}_{p} \mathcal{M}_{1}+\mathcal{M}_{p} \mathcal{M}_{2}\right)\|u\|_{o \mathcal{W}_{2}^{3}} \\
& +\left(\mathcal{M}_{p} \mathcal{M}_{2}+\mathcal{M}_{p} \mathcal{M}_{3}\right)\|u\|_{o \mathcal{W}_{2}^{3}} \\
& =\mathcal{M}_{p}\left(\mathcal{M}_{1}+\mathcal{M}_{2}+\mathcal{M}_{3}\right)\|u\|_{o \mathcal{W}_{2}^{3}}
\end{aligned}
$$

where $\mathcal{M}_{1}>0, \mathcal{M}_{2}>0, \mathcal{M}_{3}>0, \mathcal{M}_{p}>0$. Thus, we get

$$
\left[(\mathcal{L} y)^{\prime}(x)\right]^{2} \leq \mathcal{M}_{p}^{2}\left(\mathcal{M}_{1}+\mathcal{M}_{2}+\mathcal{M}_{3}\right)^{2}\|u\|_{o \mathcal{W}_{2}^{3}}^{2}
$$

and

$$
\int_{0}^{1}\left[(\mathcal{L} u)^{\prime}(x)\right]^{2} \mathrm{~d} x \leq \mathcal{M}_{p}^{2}\left(\mathcal{M}_{1}+\mathcal{M}_{2}+\mathcal{M}_{3}\right)^{2}\|u\|_{{ }^{2} \mathcal{W}_{2}^{3}}^{2},
$$


that is

$$
\begin{aligned}
& \|\mathcal{L} y\|_{\mathcal{W}_{2}^{1}}^{2}=\int_{0}^{1}\left[[(\mathcal{L} u)(x)]^{2}+\left[(\mathcal{L} u)^{\prime}(x)\right]^{2}\right] \mathrm{d} x \\
& \leq\left(\mathcal{M}_{1}+\mathcal{M}_{2}\right)^{2} \mathcal{M}_{p}^{2}\|u\|_{{ }^{\prime} \mathcal{W}_{2}^{3}}^{2} \\
& +\mathcal{M}_{p}^{2}\left(\mathcal{M}_{1}+\mathcal{M}_{2}+\mathcal{M}_{3}\right)^{2}\|u\|_{o \mathcal{W}_{2}^{3}}^{2} \\
& =\mathcal{M}_{p}^{2}\left(\left(\mathcal{M}_{1}+\mathcal{M}_{2}+\mathcal{M}_{3}\right)^{2}+\left(\mathcal{M}_{1}+\mathcal{M}_{2}\right)^{2}\right)\|u\|_{{ }^{\prime} \mathcal{W}_{2}^{3}}^{2} \\
& =\mathcal{M}\|u\|_{{ }^{\prime} \mathcal{W}_{2}^{3}}^{2}
\end{aligned}
$$

where $\mathcal{M}=\mathcal{M}_{p}^{2}\left(\left(\mathcal{M}_{1}+\mathcal{M}_{2}+\mathcal{M}_{3}\right)^{2}+\left(\mathcal{M}_{1}+\right.\right.$ $\left.\left.\mathcal{M}_{2}\right)^{2}\right)>0$.

\subsection{Solutions of the problems}

Obviously, defined operator in (9) as $\mathcal{L}$ : ${ }^{o} \mathcal{W}_{2}^{3}[0,1] \rightarrow \mathcal{W}_{2}^{1}[0,1]$ is a bounded linear operator.

Let us define $\varphi_{i}(x)=T_{x_{i}}(x)$ and $\psi_{i}(x)=\mathcal{L}^{*} \varphi_{i}(x)$, where $\mathcal{L}^{*}$ is conjugate operator of $\mathcal{L}$. The orthonormal system $\left\{\hat{\psi}_{i}(x)\right\}_{1}^{\infty} \subseteq{ }^{o} \mathcal{W}_{2}^{3}[0,1]$ can be attained by the Gram-Schmidt orthogonalization process of $\left\{\psi_{i}(x)\right\}_{1}^{\infty}$ :

$$
\hat{\psi}_{i}(x)=\sum_{k=1}^{i} \beta_{i k} \psi_{k}(x),\left(\beta_{i i}>0, i=1,2, \ldots\right) .
$$

Theorem 3. If $y(x)$ is the exact solution of (10), then

$$
y(x)=\sum_{i=1}^{\infty} \sum_{k=1}^{i} \beta_{i k} \mathfrak{f}\left(x_{k}, y_{k}\right) \hat{\psi}_{i}(x),
$$

where $\left\{x_{i}\right\}_{1}^{\infty}$ is dense in $[0,1]$.

Proof. By the (12) and uniqueness of solution of (10) we obtain:

$$
\begin{aligned}
y(x) & =\sum_{i=1}^{\infty}\left\langle y(x), \hat{\psi}_{i}(x)\right\rangle_{{ }_{o} \mathcal{W}_{2}^{3}} \hat{\psi}_{i}(x) \\
& =\sum_{i=1}^{\infty} \sum_{k=1}^{i} \beta_{i k}\left\langle y(x), \psi_{k}(x)\right\rangle_{o \mathcal{W}_{2}^{3}} \hat{\psi}_{i}(x) \\
& =\sum_{i=1}^{\infty} \sum_{k=1}^{i} \beta_{i k}\left\langle y(x), \mathcal{L}^{*} \varphi_{k}(x)\right\rangle_{{ } \mathcal{W}_{2}^{3}} \hat{\psi}_{i}(x) \\
& =\sum_{i=1}^{\infty} \sum_{k=1}^{i} \beta_{i k}\left\langle\mathcal{L} y(x), \varphi_{k}(x)\right\rangle_{\mathcal{W}_{2}^{1}} \hat{\psi}_{i}(x) \\
& =\sum_{i=1}^{\infty} \sum_{k=1}^{i} \beta_{i k}\left\langle\mathfrak{f}(x, y), T_{x_{k}}\right\rangle_{\mathcal{W}_{2}^{1}} \hat{\psi}_{i}(x) \\
& =\sum_{i=1}^{\infty} \sum_{k=1}^{i} \beta_{i k} \mathfrak{f}\left(x_{k}, y_{k}\right) \hat{\psi}_{i}(x) .
\end{aligned}
$$

Finite terms of (13) concludes the approximate solution:

$$
y_{n}(x)=\sum_{i=1}^{n} \sum_{k=1}^{i} \beta_{i k} \mathfrak{f}\left(x_{k}, y_{k}\right) \hat{\psi}_{i}(x) .
$$

Lemma 1. If $\left\|y_{n}-y\right\|_{o \mathcal{W}_{2}^{3}} \rightarrow 0, x_{n} \rightarrow x,(n \rightarrow$ $\infty)$ and $\mathfrak{f}(x, y)$ is continuous w.r.t. $x \in[0,1]$, then

$$
\mathfrak{f}\left(x_{n}, y_{n-1}\left(x_{n}\right)\right) \rightarrow \mathfrak{f}(x, u(x)), \quad \text { as } n \rightarrow \infty .
$$

Theorem 4. Let for any fixed $y_{0}(x) \in{ }^{o} \mathcal{W}_{2}^{3}[0,1]$ we have

(i)

$$
y_{n}(x)=\sum_{i=1}^{n} A_{i} \hat{\psi}_{i}(x)
$$

where

$$
A_{i}=\sum_{k=1}^{i} \beta_{i k} \mathfrak{f}\left(x_{k}, y_{k-1}\left(x_{k}\right)\right),
$$

(ii) $\left\|y_{n}\right\|_{o \mathcal{W}_{2}^{3}}$ is bounded;

(iii) $\left\{x_{i}\right\}_{1}^{\infty}$ is dense in $[0,1]$;

(iv) $\mathfrak{f}(x, y) \in \mathcal{W}_{2}^{1}[0,1]$ for any $y(x) \in$ ${ }^{\circ} \mathcal{W}_{2}^{3}[0,1]$. Then the approximate solution $y_{n}(x)$ converges to the exact solution of (13) in ${ }^{\circ} \mathcal{W}_{2}^{3}$ and we have

$$
y(x)=\sum_{i=1}^{\infty} A_{i} \hat{\psi}_{i}(x) .
$$

Proof. First, we prove the convergence of $y_{n}(x)$. From (15), we have

$$
y_{n+1}(x)=y_{n}(x)+A_{n+1} \hat{\psi}_{n+1}(x) .
$$

Also, orthonormality of $\left\{\hat{\psi}_{i}\right\}_{i=1}^{\infty}$, yields

$$
\begin{aligned}
& \left\|y_{n+1}\right\|_{{ }^{\circ} \mathcal{W}_{2}^{3}}^{2}=\left\|y_{n}\right\|_{{ }^{\circ} \mathcal{W}_{2}^{3}}^{2}+A_{n+1}^{2} \\
& =\left\|y_{n-1}\right\|_{{ }^{\circ} \mathcal{W}_{2}^{3}}^{2}+A_{n}^{2}+A_{n+1}^{2}=\ldots=\sum_{i=1}^{n+1} A_{i}^{2},
\end{aligned}
$$

and from boundedness of $\left\|y_{n}\right\|_{o_{\mathcal{W}_{2}^{3}}^{3}}$, we obtain

$$
\sum_{i=1}^{\infty} A_{i}^{2}<\infty
$$

i.e.,

$$
\left\{A_{i}\right\} \in l^{2} \quad(i=1,2, \ldots) .
$$

Let $m>n$, in view of $\left(y_{m}-y_{m-1}\right) \perp\left(y_{m-1}-\right.$ $\left.y_{m-2}\right) \perp \ldots \perp\left(y_{n+1}-y_{n}\right)$, we get 


$$
\begin{aligned}
& \left\|y_{m}-y_{n}\right\|_{o \mathcal{W}_{2}^{3}}^{2}=\| y_{m}-y_{m-1}+y_{m-1}-y_{m-2} \\
& +\ldots+y_{n+1}-y_{n} \|_{o \mathcal{W}_{2}^{3}}^{2} \\
& =\left\|y_{m}-y_{m-1}\right\|_{o \mathcal{W}_{2}^{3}}^{2}+\ldots+\left\|y_{n+1}-y_{n}\right\|_{o \mathcal{W}_{2}^{3}}^{2} \\
& =\sum_{i=n+1}^{m} A_{i}^{2} \rightarrow 0, \quad m, n \rightarrow \infty .
\end{aligned}
$$

Considering the completeness of ${ }^{o} \mathcal{W}_{2}^{3}[0,1]$, there exists $y(x) \in{ }^{o} \mathcal{W}_{2}^{3}[0,1]$, such that

$$
y_{n}(x) \rightarrow y(x) \quad \text { as } n \rightarrow \infty .
$$

(ii) Now, we show $y(x)$ is the exact solution of (10). Tacking limits in (15) we get

$$
y(x)=\sum_{i=1}^{\infty} A_{i} \hat{\psi}_{i}(x) .
$$

Thus, we reach

$$
(\mathcal{L} y)\left(x_{2}\right)=\mathfrak{f}\left(x_{2}, y_{1}\left(x_{2}\right)\right) .
$$

Moreover, by induction we conclude

$$
(\mathcal{L} y)\left(x_{j}\right)=\mathfrak{f}\left(x_{j}, y_{j-1}\left(x_{j}\right)\right) .
$$

From $\overline{\left\{x_{i}\right\}_{i=1}^{\infty}}=[0,1]$, it can be presented that for any $\xi \in[0,1]$, there exists $\left\{x_{n_{j}}\right\}_{1}^{\infty} \subseteq\left\{x_{i}\right\}_{1}^{\infty}$, such that $\lim _{j \rightarrow \infty} x_{n_{j}}=\xi$. Therefore, convergence of $y_{n}(x)$ and Lemma 4.3 yields

$$
(\mathcal{L} y)(\xi)=\mathfrak{f}(\xi, y(\xi))
$$

So, $y(x)$ is the exact solution of (10) given by

$$
y(x)=\sum_{i=1}^{\infty} A_{i} \hat{\psi}_{i}(x),
$$

where $A_{i}$ are given by (16).

Theorem 5. If $y \in{ }^{o} \mathcal{W}_{2}^{3}[0,1]$ then

$$
\left\|y_{n}-y\right\|_{o \mathcal{W}_{2}^{3}} \rightarrow 0, \quad n \rightarrow \infty .
$$

Moreover a sequence $\left\|y_{n}-y\right\|_{o_{\mathcal{W}_{2}^{3}}}$ is monotonically decreasing in $n$.

\section{Proof.}

By (13) and (14), we acquire

$$
\left\|y_{n}-y\right\|_{{ }_{\mathcal{W}_{2}^{3}}}=\left\|\sum_{i=n+1}^{\infty} \sum_{k=1}^{i} \beta_{i k} \mathfrak{f}\left(x_{k}, y_{k}\right) \hat{\psi}_{i}\right\|_{{ }_{\mathcal{W W}_{2}^{3}}} .
$$

Therefore, we obtain

$$
\left\|y_{n}-y\right\|_{{ }_{\mathcal{W}}^{3}} \rightarrow 0, \quad n \rightarrow \infty .
$$

In addition

$$
\begin{gathered}
\left\|y_{n}-y\right\|_{\mathcal{W}_{2}^{3}}^{2}=\left\|\sum_{i=n+1}^{\infty} \sum_{k=1}^{i} \beta_{i k} \mathfrak{f}\left(x_{k}, y_{k}\right) \hat{\psi}_{i}\right\|_{o \mathcal{W}_{2}^{3}}^{2} \\
=\sum_{i=n+1}^{\infty}\left(\sum_{k=1}^{i} \beta_{i k} \mathfrak{f}\left(x_{k}, y_{k}\right) \hat{\psi}_{i}\right)^{2} .
\end{gathered}
$$

\section{Numerical examples}

We consider the following problems by reproducing kernel Hilbert space method in this section. We computed our results by MAPLE. We showed our results by tables.

Example 1. We investigate:

$$
\begin{gathered}
\left(\frac{1}{1+t} u(t)^{\prime}\right)^{\prime}=2 \exp (3 u(t)) \quad 0 \leq t \leq 1 \\
u(0)=0 \quad u(1)=-\log _{e}(2)
\end{gathered}
$$

We have the exact solution of the above problem as:

$$
u(t)=\log _{e}(1 /(1+t)) .
$$

We searched the boundary value problem (20) by the proposed method and gave corresponding error-norms by Table 1 .

Example 2. We solved the following problem for the second example in the reproducing kernel Hilbert space.

$$
\left(\left(1+t^{2}\right) u(t)^{\prime}\right)^{\prime}-\left(1+t-t^{2}\right) u(t)=h(t)
$$

$$
u(0)=0 \quad u(1)=0
$$

We get the exact solution of the above problem as:

$$
u(t)=1+(t-1) \exp (-t)-t \exp (-(1-t)) .
$$

In Table 2, we computed absolute errors for (21).

Table 1. Maximum absolute errors (MAE) of the first example.

\begin{tabular}{ccc}
\hline & $N=64, \sigma=1.02$ & $N=64, \sigma=1.02$ \\
\hline RKHSM & $5.46 E-12$ & $5.46 E-12$ \\
{$[10]$} & $1.70 E-08$ & $2.85 E-06$ \\
{$[11]$} & $8.49 E-04$ & $1.21 E-02$ \\
11 & $2.43 E-03$ & $1.88 E-02$ \\
113 & $5.63 E-03$ & $2.70 E-02$ \\
\hline
\end{tabular}


Table 2. Maximum absolute errors (MAE) of the second example.

\begin{tabular}{ccc}
\hline & $N=64, \sigma=1.02$ & $N=64, \sigma=1.02$ \\
\hline RKHSM & $9.20 E-11$ & $9.20 E-11$ \\
{$[10]$} & $8.07 E-06$ & $6.09 E-05$ \\
{$[11]$} & $1.86 E-03$ & $1.08 E-02$ \\
{$[12]$} & $2.16 E-03$ & $9.87 E-03$ \\
{$[13]$} & $5.19 E-04$ & $2.64 E-03$ \\
\hline
\end{tabular}

\section{Conclusions}

In this work, we gave a new application of the reproducing kernel Hilbert space method. We obtained very useful reproducing kernel functions in the reproducing kernel Hilbert spaces. We proved the accuracy of the method. We compared the reproducing kernel Hilbert space method with the techniques existed in the literature. We concluded that the proposed technique is very effective for solving nonlinear two-point boundary value problems.

\section{References}

[1] Safari, M.J.S., Rahimzadeh Arashloo, S. \& Danandeh, M. (2020). A. Rainfall-runoff modeling through regression in the reproducing kernel Hilbert space algorithm. Journal of Hydrology 587, 125014.

[2] Najafi, N. \& Allahviranloo, T. (2020). Combining fractional differential transform method and reproducing kernel Hilbert space method to solve fuzzy impulsive fractional differential equations. Computational and $A p$ plied Mathematics. 39(2), 122.

[3] Sahihi, H., Allahviranloo, T. \& Abbasbandy, S. (2020). Solving system of second-order BVPs using a new algorithm based on reproducing kernel Hilbert space. Applied Numerical Mathematics, 151, pp. 27-39.

[4] Agud, L., Calabuig, J.M. \& Sánchez Pérez, E.A. (2020). Weighted p -regular kernels for reproducing kernel Hilbert spaces and Mercer Theorem. Analysis and Applications, 18(3), pp. 359-383.

[5] Mundayadan, A. \& Sarkar, J. (2020). Linear dynamics in reproducing kernel Hilbert spaces. Bulletin des Sciences Mathematiques, 159, 2020102826.

[6] Abu Arqub, O. \& Maayah, B. (2019). Modulation of reproducing kernel Hilbert space method for numerical solutions of Riccati and Bernoulli equations in the Atangana-Baleanu fractional sense. Chaos, Solitons and Fractals, $125,163-170$.
[7] Emamjome, M., Azarnavid, B. \& Ghehsareh, H.R. (2019). A reproducing kernel Hilbert space pseudospectral method for numerical investigation of a two-dimensional capillary formation model in tumor angiogenesis problem. Neural Computing and Applications, 31(7), 2233-2241.

[8] Foroutan, M., Asadi, R. \& Ebadian, A. (2019). A reproducing kernel Hilbert space method for solving the nonlinear three-point boundary value problems. International Journal of Numerical Modelling: Electronic Networks, Devices and Fields, 32(3), e2573.

[9] Karatas Akgül, E., Akgül, A., Khan, Y. \& Baleanu, D. (2019). Representation for the reproducing kernel hilbert space method for a nonlinear system. Hacettepe Journal of Mathematics and Statistics, 48(5), 1345-1355.

[10] Jain, M.K., Sharma, S. \& Mohanty, R.K. (2016). High accuracy variable mesh method for nonlinear two-point boundary value problems in divergence form. Applied Mathematics and Computation, 273, 885-896.

[11] Mohanty, R.K. (2005). A family of variable mesh methods for the estimates of $(\mathrm{du} / \mathrm{dr})$ and solution of non-linear two point boundary value problems with singularity. Journal of Computational and Applied Mathematics, 182, 173-187.

[12] Mohanty, R.K., Evans, D.J. \& Khosla, N. (2005). An $O\left(h_{k}^{3}\right)$ non-uniform mesh cubic spline TAGE method for non-linear singular two-point boundary value problems. International Journal of Computer Mathematics, 82, $1125-1139$.

[13] Mohanty, R.K. \& Khosla, N. (2006). Application of TAGE iterative algorithms to an efficient third order arithmetic average variable mesh discretization for two-point non-linear boundary value problems. Applied Mathematics and Computation, 172 148-162.

[14] Allahviranloo, T. \& Sahihi, H. (2020). Reproducing kernel method to solve parabolic partial differential equations with nonlocal conditions. Numerical Methods for Partial Differential Equations, 36(6), 1758-1772.

[15] Abu Arqub, O. \& Al-Smadi, M. (2018). Atangana-Baleanu fractional approach to the solutions of Bagley-Torvik and Painlevé equations in Hilbert space. Chaos, Solitons and Fractals, 117, 161-167.

[16] Abu Arqub, O. \& Maayah, B. (2018). Numerical solutions of integrodifferential equations of Fredholm operator type in the sense of the Atangana-Baleanu fractional operator. Chaos, Solitons and Fractals, 117, 117-124. 
[17] Abu Arqub, O. \& Maayah, B. (2019). Fitted fractional reproducing kernel algorithm for the numerical solutions of ABC - Fractional Volterra integro-differential equations. Chaos, Solitons and Fractals, 126, 394-402.

[18] Abu Arqub, O. \& Maayah, B. (2019). Modulation of reproducing kernel Hilbert space method for numerical solutions of Riccati and Bernoulli equations in the Atangana-Baleanu fractional sense. Chaos, Solitons and Fractals, 125, 163-170.

[19] Abu Arqub, O. \&, Al-Smadi, M. (2020). Fuzzy conformable fractional differential equations: novel extended approach and new numerical solutions. Soft Computing, 24, 12501-12522.

[20] Yavuz, M. \& Evirgen, F. (2018). An alternative approach for nonlinear optimization problem with Caputo-Fabrizio derivative. In ITM Web of Conferences, Vol.22, p.01009.

[21] Yavuz, M. \& Ozdemir, N. (2018). On the solutions of fractional Cauchy problem featuring conformable derivative. In ITM Web of Conferences, Vol.22, p.01045.
[22] Evirgen, F. (2016). Analyze the optimal solutions of optimization problems by means of fractional gradient based system using VIM. An International Journal of Optimization and Control: Theories 85 Applications (IJOCTA), 6(2), 75-83.

[23] Yavuz, M. \& Sene, N. (2020). Approximate solutions of the model describing fluid flow using generalized -Laplace transform method and heat balance integral method. Axioms, $9(4), 123$.

Ali Akgül is an associate professor at Siirt University, Turkey. He has many publications on fractional calculus and numerical methods.

(10)https://orcid.org/0000-0001-9832-1424

Mir Sajjad Hashemi is an associate professor at Bonab University, Iran. He has many publications on fractional calculus and numerical methods.

(10https://orcid.org/0000-0002-5529-3125

Negar Seyfi is a researcher from Bonab University, Iran.

(10https://orcid.org/0000-0002-1760-0745

An International Journal of Optimization and Control: Theories \& Applications (http://ijocta.balikesir.edu.tr)

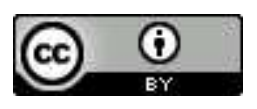

This work is licensed under a Creative Commons Attribution 4.0 International License. The authors retain ownership of the copyright for their article, but they allow anyone to download, reuse, reprint, modify, distribute, and/or copy articles in IJOCTA, so long as the original authors and source are credited. To see the complete license contents, please visit http://creativecommons.org/licenses/by/4.0/. 